\title{
Central corneal thickness, keratometry and intra ocular pressure measurement in a case control comparative study between pseudoexfoliative (PXF) to non pseudoexfoliative cataract patients
}

\author{
Manoj Kumar ${ }^{1}$, Soumyakanta Mohanty²*
}

${ }_{1}^{1}$ IIrd Year Resident, Department of Ophthalmology, Kalinga Institute of Medical Sciences and Pradyumna Bal Memorial Hospital, Bhubaneswar, Odisha, 751024, INDIA.

Email: godjagannath74@gmail.com

\begin{abstract}
Background: PXF is a degenerative condition with the deposition of PXF (pseudoexfoliative materials) all over the different organs of the body. It is also found predominantly in the anterior segment of the eye. It causes moderate to severe complications in cataract surgery and cause blinding diseases like glaucoma. PXF affects the anatomical and functional components of the eye. Methods: 70 patients of PXF and 70 patients of non PXF (including either sex) over 50 years of age with cataract were evaluated for CCT (central corneal thickness), keratometry and IOP (intra ocular pressure). PXF with glaucoma was excluded from the study. Results: The CCT was $500 \mu \mathrm{m}$ in PXF patients as that to $540 \mu \mathrm{m}$ in non PXF patients. Hence the corrected IOP after correcting for decreased CCT was $18 \pm 3 \mathrm{~mm}$ of $\mathrm{Hg}$ in PXF patients, whereas there was not much difference in the corrected and uncorrected IOP in non PXF patients. The keratometry was normal in both PXF and non PXF patients. Conclusion: The CCT was generally found decreased in PXF patients. Hence, corrected IOP should be done to prevent inadvertent complications due to falsely decreased IOP.

Key words: Pseudoexfoliation, CCT, IOP, keratometry.
\end{abstract}

*Address for Correspondence:

Dr Soumyakanta Mohanty, Department of Ophthalmology, Kalinga Institute of Medical Sciences and Pradyumna Bal Memorial Hospital, Bhubaneswar, Plot-287, Unit-3 kharvelanagar Bhubaneswar, Odisha, INDIA.

Email: godjagannath74@gmail.com

Received Date: 02/12/2019 Revised Date: 15/01/2020 Accepted Date: 07/02/2020

DOI: https://doi.org/10.26611/10091511

This work is licensed under a Creative Commons Attribution-NonCommercial 4.0 International License. (cc)) BY-NC

\begin{tabular}{|l|l|}
\hline \multicolumn{2}{|c|}{ Access this article online } \\
\hline Quick Response Code: & Website: \\
\hline & www.medpulse.in \\
& \\
\hline
\end{tabular}

\section{INTRODUCTION}

PXF is generally termed as pseudoexfoliation to differentiate from true exfoliation. Pseudoexfoliation has been in wrong light due to its accidental or elective ocular complication. PXF could affect the ocular structure in all ways and segments. Notorious in its outcome PXF has been mostly found in structures like trabecular meshwork, iris, lens, ciliary body and anterior and posterior segment also. The most distinctive outcome of PXF pathogenesis is its way of blinding disease namely complication of cataract surgery and glaucoma. Generally, it causes secondary high tension glaucoma, mostly open angle type. Cataract morbidity could be limited or leads to disastrous complications. So identification of PXF in all its manifestation could salvage the eyes and the individual's quality of life. PXF generally senile markedly increases with age, racial differentiation exists from $0-25 \% .{ }^{1,2}$ Lots of literatures have been given by different studies for the known complications of PXF. This study evaluates the corneal status and effect of PXF on the cornea preoperatively. Corneal edema and increased corneal thickness are a known post cataract surgery with PXF 
complication. ${ }^{3}$ Hence we in this study evaluate the corneal thickness preoperatively during biometry and cataract evaluation. We compare this to the existing literatures worldwide as well as establish the relationship in eastern Indian local population. The comparison of central corneal thickness in senile cataract males and females with or without PXF could give us a more detailed understanding of PXF, cataract and central corneal thickness from its preoperative and expected later post-operative surgical prognosis.

\section{MATERIAL AND METHODS}

This study has been done according to the tenets of the declaration of Helsinki. It is hospital based case control observational comparative study. A group of 70 PXF positive patients both males and females termed as cases were compared with a control of 70 non PXF patients. The patients were included for the study after detailed history and examinations. Prior to the study a written consent was taken of all patients willing to participate in this study. All the preoperative cataract evaluation was done; after a previous detailed medical history was taken. The biometry, IOL calculation, intraocular pressure (applanation tonometry) and preoperative detailed cataract evaluation was done. The central corneal thickness was taken in the eye to be operated for cataract with PXF and was compared with normal population with cataract but without PXF. IOL master was used for finding out central corneal thickness. Other parameters such as K1, K2 (keratometry), axial length, anterior chamber depth were also calculated in both groups. Intra ocular pressure was also noted. Preoperative blood and local investigations was also carried out including blood pressure to rule out hypertension etc. IOL master evaluation prior to cataract surgery is a noncontact technique and done with utmost accuracy and care. The patients were operated for cataract surgery in both groups with manual small incision cataract surgery. Cases of high intra ocular pressure were noted and preoperative evaluation was done for glaucoma diagnosis. Other glaucoma diagnostic examinations namely $+90 \mathrm{D}$ funduscopy, gonioscopy, OCT- RNFL was also done. The patients diagnosed with glaucoma were excluded from the study.

\section{Inclusion criteria}

All patients more than 50 years' males and females, with or without PXF, after detailed cataract and fundus evaluation were included.

Exclusion criteria

All patients less than 50 years, traumatic cataract, exposed to infrared light, other ocular diseases, except PXF, uncontrolled diabetes, hypertension and cardiovascular disease and cataract with unwilling to participate in this study were excluded.

Ethical approval taken from Institutions ethical committee.

\section{RESULT}

After a study of one and half years, the data was assembled and the following result was found.

The average age of non PXF patients was $67.54 \pm 8.5$ (SD) years with 33 females and 37 males. In the PXF group the average age was $69.12 \pm 8.4(\mathrm{SD})$ years with 31 females and 39 males. Evaluation of corneal measurement by IOL master concluded that keratometry reading was not statistically significantly different in both groups (PXF and non PXF group).

The K1 (horizontal meridian) keratometry was $44.20 \pm$ 1.13 (SD) D in non PXF whereas in PXF it was $44.06 \pm$ 1.35 (SD) D. The K2 (vertical meridian) keratometry was $44.50 \pm 0.91(\mathrm{SD}) \mathrm{D}$ in non PXF whereas in PXF it was $44.30 \pm 0.8$ (SD) D. Both K1 and K2 was statistically not significantly different. The CCT was significantly different in both groups with the non PXF having a CCT of 546.57 \pm 2.83 (SD) $\mu \mathrm{m}$ whereas the PXF group was $500.14 \pm 3.75$ (SD) $\mu \mathrm{m}$. This suggests that PXF group have a significantly thinner cornea to that of the normal counterpart. The IOP in both groups did not show a significant difference. The IOP in uncorrected state (regarding correcting factor to $\mathrm{CCT}$ ) the non PXF group had an IOP of $15.14 \pm 2.5$ (SD) $\mathrm{mmHg}$ whereas in the PXF group was $15.64 \pm 2.96(\mathrm{SD}) \mathrm{mmHg}$. This difference was not statistically significant. But after the correction of IOP based on correction factor due to decrease or increased CCT, the corrected IOP in non PXF group was $15.37 \pm$ 2.85 (SD) $\mathrm{mmHg}$ whereas in the PXF group was $18.77 \pm$ 3.86 (SD) $\mathrm{mmHg}$. This difference is statistically significant with $\mathrm{P}$ value $<0.0001$.

Table 1: Statistically comparative data between PXF and non PXF groups

\begin{tabular}{|c|c|c|c|c|c|c|}
\hline & $\begin{array}{l}\text { Age (Years) } \\
{[\text { Mean } \pm \text { SD] }}\end{array}$ & $\begin{array}{c}\text { CCT }(\mu \mathrm{m}) \\
{[\text { Mean } \pm \mathrm{SD}]}\end{array}$ & $\begin{array}{c}\text { K1 (D) } \\
{[\text { Mean } \pm \text { SD] }}\end{array}$ & $\begin{array}{c}\text { K2 (D) } \\
{[\text { Mean } \pm \text { SD] }}\end{array}$ & $\begin{array}{c}\text { Uncorrected IOP }(\mathrm{mm} \text { of } \mathrm{Hg}) \\
{[\text { Mean } \pm \mathrm{SD}]}\end{array}$ & $\begin{array}{c}\text { Corrected IOP }(\mathrm{mm} \text { of } \mathrm{Hg}) \\
{[\text { Mean } \pm \text { SD }}\end{array}$ \\
\hline Non PXF & $\begin{array}{c}67.54 \pm 8.5 \\
(33-F \text { and } 37-M)\end{array}$ & $546.57 \pm 2.83$ & $44.20 \pm 1.13$ & $44.50 \pm 0.9$ & $15.14 \pm 2.5$ & $15.37 \pm 2.8$ \\
\hline PXF & $\begin{array}{c}69.12 \pm 8.4 \\
(31-F \text { and } 39-M)\end{array}$ & $500.14 \pm 3.75$ & $44.06 \pm 1.35$ & $44.30 \pm 0.8$ & $15.64 \pm 2.96$ & $18.77 \pm 3.8$ \\
\hline P-value & $<0.2364$ & $<0.0001$ & $<0.5015$ & $<0.3216$ & $<0.2828$ & $<0.0001$ \\
\hline
\end{tabular}

The males and females have no different parameters in both groups. Both males and females have distinctively decreases central corneal thickness in PXF patients as compared to age and sex in the other group of non PXF. 


\section{DISCUSSION}

Central corneal thickness in different individuals varies according to population, race and gender. Such as, so PXF is found in nearly all races more or less. The relationship between PXF and CCT has been quite controversial. PXF causes glaucoma associated with hard nuclear cataract and causes cataract complications in larger numbers than in senile cataract without PXF. CCT have a role in estimation of IOP, with decreasing CCT resulting in falsely low IOP and increasing CCT resulting in falsely high IOP. ${ }^{4}$ Thus the normal CCT in Indian scenario has been calculated as around $540 \mu \mathrm{m}$. Increased CCT needs for a negative correction factor as well as decrease CCT needs a positive correction factor. Thus it's necessary to have a corrected IOP after CCT evaluation for a more accurate IOP. Conditions of IOP measured aberrantly can give to disastrous consequences most likely in anterior segment surgeries. Similarly, in CCT measured aberrantly may lead to disastrous consequences in ocular surgery. ${ }^{5}$ Study by Georgious Kitsos shows that CCT in PXF glaucoma was $(526 \pm 34.3 \mu \mathrm{m})$, the CCT in PXF without glaucoma was $550.64 \pm 39 \mu \mathrm{m}$ and $547.36 \pm 33.1 \mu \mathrm{m}$ in control group without PXF. ${ }^{6}$ This study shows no difference between patients with PXF and without PXF. Only in cases of PXF glaucoma the CCT is decreased which could mean a progressive PXF syndrome causes decreased CCT with glaucoma development. Study by Ozcura shows that CCT was decreased to that of normal control eyes and PXF glaucoma had thicker or same thickness of CCT as compare to normal eyes. ${ }^{7}$ But keratometry was same in both PXF groups with or without glaucoma and normal control eyes. Combination of CCT and IOP measurements could lead to problems post operatively if not evaluated correctly. Thus in this study we have found that CCT definitely has a lower caliber or is thinner than the general population. The averages mean being $500.14 \pm 3.75 \mu \mathrm{m}$ in PXF patients to that $546 \pm 2.83 \mu \mathrm{m}$ in general populations. Such being the case, the comparison of CCT in other population could derive us to conclusion of variation and association of PXF to other ocular (intra and extra- ocular) structures. In study (Pseudoexfoliation in the Reykjavik eye study) showed that PXF doesn't alter the CCT in all groups and was normal as per eyes without PXF. ${ }^{8}$ This study is conducted in white race populations. In another study Ebrahim et al. concluded that PXF patients had significantly steeper corneal than normal patients without PXF. ${ }^{9}$ In contrast to our study, Ibrahim's patients with PXF glaucoma had a higher CCT than normal eyes without PXF. Thus it concluded that overestimation of IOP than the true IOP may occur in such eyes. This study was done in Turkey populations. The keratometry finding in our study doesn't show the statistical difference between the PXF and non PXF groups. Some studies have shown that cornea is steep in PXF glaucoma cases than normal populations without PXF. The IOP was not different statistically between both PXF and non PXF groups. After standard correction for IOP depending on the thicker and thinner cornea, we found that PXF cases having thinner cornea had a greater rise of corrected IOP than the non PXF group with thicker cornea. Thus corrected IOP is necessary for evaluation of true IOP as if not corrected it will give a falsely lower IOP which is detrimental for anterior segment surgeries. IOP measured by applanation tonometry has proved that due to decrease CCT the corrected IOP has a higher IOP with almost +1 to +7 mmHg. Clarifying the context, does CCT has a dependence on PXF. The CCT of normal patients doesn't have a lower range than PXF patients; and a formidable change in all case of decreased CCT with PXF definitely could be assumed to be a dependent variable or association. Other structures of eyeball namely retina, choroid, sclera evaluation could open a well wide range of possibilities. Further studies on this aspect could be more illuminating and such research could reveal more secrets of ophthalmological research and the eyes in general. Our study suggests a falsely decreased IOP in PXF patients due to thinner cornea, hence after corrections of IOP in considerations to decreased CCT there is increased IOP. Thus, evaluation of CCT is necessary as we have variable IOP which could be increased or decreased in different geographical populations and race. IOP after standard corrections shows that in increased CCT, there is corrected decreased IOP. Whereas, in decreased CCT, there is corrected increased IOP.

\section{CONCLUSION}

IOP should always be measured with correction done in relation to CCT as decreased CCT in PXF can give rise to falsely low IOP which could lead to disastrous consequences. The CCT in PXF should always be measured as thin corneas are more vulnerable in anterior segment surgeries. In short proper careful and detailed evaluation of the structures (cornea) should be done to prevent any unwanted events during or post anterior segment surgeries.

\section{REFERENCES}

1. Nilgun Yildirim, Erdogan Yasar, Huseyin Gursoy, and Ertugrul Colak. Prevalence of pseudoexfoliation syndrome and its association with ocular and systemic diseases in Eskisehir, Turkey. Int J Ophthalmol. 2017; 10(1): 128-134.

2. Schlötzer-Schrehardt, Naumann GO. Ocular and systemic pseudoexfoliation syndrome. Am J Ophthalmol. 2006 May; 141(5):921-937.

3. K Pranathi, Renu M Magdum, Rupali Maheshgauri, Khevna Patel, S Patra. A study of complications during 
cataract surgery in patients with pseudoexfoliation syndrome. Journal of clinical ophthalmology and research. 2014; 2 (1): 7-11.

4. Prasanta Kumar Nanda1, Bishnupriya Khuntia2, Shradha Pattnaik. Diurnal Variation of Central Corneal Thickness (CCT) and Intraocular Pressure (IOP) in Subjects with Pseudoexfoliation Glaucoma, Pseudoexfoliation Syndrome and in Normal Subjects. J. Evid. Based Med. Healthc. 2020; 7(1); P-2349-2562.

5. Nikhil S Choudhari, Ronnie George, Ramesh Ve Sathyamangalam, Prema Raju, Rashima Asokan, Lokapavani Velumuri, Lingam Vijaya. Long-term change in central corneal thickness from a glaucoma perspective. 2013; 61 (10); 580-584.

6. Georgios Kitsos, Christos Gartzios, Ioannis Asproudis, and Eleni Bagli. Central corneal thickness in subjects with glaucoma and in normal individuals (with or without pseudoexfoliation syndrome. Clin Ophthalmol. 2009; 3: 537-542

7. Ozcura F, Aydin S, Dayanir V. Central corneal thickness and corneal curvature in pseudoexfoliation syndrome with and without glaucoma. J Glaucoma. 2011 Sep; 20(7):4103.

8. Arsaell Arnarsson Karim F. Damji HiroshiSasaki Thordur Sverrisson Fridbert Jonasson. Pseudoexfoliation in the Reykjavik Eye Study: Five-Year Incidence and Changes in Related Ophthalmologic Variables. American Journal of Ophthalmology.2009; 148(2); 291-297.

9. Ibrahim F Hepsen, Ramazan YağcI, Urğcan Keskin. Corneal curvature and central corneal thickness in eyes with pseudoexfoliation syndrome. Canadian journal of ophthalmology.2007; 42(5); P677-680.

\section{Source of Support: None Declared Conflict of Interest: None Declared}

\section{Policy for Articles with Open Access:}

Authors who publish with MedPulse International Journal of Ophthalmology (Print ISSN: 2250-7575) (Online ISSN: 2636-4700) agree to the following terms: Authors retain copyright and grant the journal right of first publication with the work simultaneously licensed under a Creative Commons Attribution License that allows others to share the work with an acknowledgement of the work's authorship and initial publication in this journal.

Authors are permitted and encouraged to post links to their work online (e.g., in institutional repositories or on their website) prior to and during the submission process, as it can lead to productive exchanges, as well as earlier and greater citation of published work. 